\title{
Die Konstitution des Ricinins
}

\author{
Von \\ Ernst Späth und Erich Tschelnitz
}

Aus dem I. Chemischen Laboratorium der Universität in Wien

(Vorgelegt in der Sitzung am 7. Juli 192l)

\section{Allgemeines.}

Das Ricinin ist das giftige Alkaloid von Ricimus commmuzis, der Ricinuspflanze, welche schon in den ältesten $Z \in i t e n$ kultiviert worden ist. Es wurde 1864 von Tuson ${ }^{1}$ zum ersten Male isoliert und später von einer Anzahl Forscher genauer untersucht. M. So ave ${ }^{2}$ ermittelte als Bruttoformel $\mathrm{C}_{47} \mathrm{H}_{18} \mathrm{~N}_{4} \mathrm{O}_{4}$, während Th. Evans ${ }^{3}$ dieselbe zu. $\mathrm{C}_{16} \mathrm{H}_{18} \mathrm{~N}_{4} \mathrm{O}_{4}$ bestimmte. Beide hielten das Ricinin für den Methylester der Ricininsäure, welche durch Verseifen von Ricinin mit Alkalien entsteht. E. Schulze und E. Winterstein ${ }^{4}$ fanden, daß das Ricinin die Weidel'sche und die Murexidreaktion gibt. Die Arbeiten von Maquenne und Philippe ${ }^{5}$ führten zur Aufstellung der Bruttoformel $\mathrm{C}_{8} \mathrm{H}_{8} \mathrm{~N}_{2} \mathrm{O}_{2}$, welche von späteren Forschern bestätigt wurde. Beim Erhitzen von Ricininsäure mit Salzsäure erhieiten diese Autoren neben Ammoniak und Kohlendioxyd einen Körper der Formel $\mathrm{C}_{6} \mathrm{H}_{7} \mathrm{NO}_{2}$, den sie als $\beta$-Oxy- $N$-methyl- $\gamma$-pyridon auffaßten. Diese Verbindung verwandelten sie mittels Phosphorpentachlorid in ein Dichlorpyridin von unbekannter. Konstitution und erhielten daraus durch Erhitzen mit Jodwasserstoffsäure Pyridin. Auf Grund dieser Umsetzungen nahmen sie für das Ricinin folgende Konstitutionsformel an:

1 Tuson, J. 1864, 457; 1870,877.

2M. Soave, B1. [3] 14, 835 (1895); Chem. Zentr. 1895, 1, 853.

"Ch. Evans, Journ. Am. Chem. Soc. 22, 39 (1900); Chem. Zentr. 1900, 1, 612.

4 E. Schulze und F. Winterstein, Zeitschr. f. physiol. Chem., 43, $211(1904)$.

5 Maquennesund Philippe, C.r. 138, 506 (1904); C.r. 139, 840 (1904). 


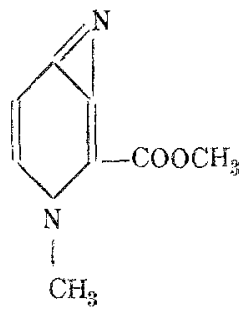

1917 erschien eine schöne Untersuchung von E. Winterstein, J. Keller und A. B. Weinhagen. Es wurde von ihnen die bemerkenswerte Tatsache berichtet, daß die reifen Samen von Ricinus commmis nur etwa $0.15 \%$, die Blätter der jungen Pflanzen aber $1.37 \%$ und die etiolierten jungen Pflänzchen nahezu $2.5 \%$ Ricinin enthalten. Beim Kochen von Ricinin mit $57 \cdot 4$ prozentiger Schwefelsäure erhielten sie auber Ammoniak und Kohlendioxyd eine Base der Formel $\mathrm{C}_{7} \mathrm{H}_{9} \mathrm{NO}_{2}$, welche noch die Methoxylgruppe des Ricinins besaB. Ricinin nahm bei Gegenwart von kolloidalem Platin 4 Atome Wasserstoff auf unter Bildung von Tetrabydroricinin. Die Formel von Maquenne und Philippe hielten sie für unrichtig. In der bald nachber erschienenen interessanten Arbeit von Bruno Böttcher ${ }^{1}$ wird die schon von Maquenne und Philippe angenommene Methylimidgruppe direkt bestimmt. Während durch Behandeln von Ricininsäure mit Methylalkohol und Salzsäure kein Ricinin zurückgewonnen werden konnte, gelang diese Umwandlung durch Behandeln von ricininsaurem Silber mit Jodmethyl. Böttcher hat durch verschiedene Reaktionen einen Glyoxalinkern im Ricinin wahrscheinlich gemacht und als Ergebnis seiner Untersuchungen die nachfolgende Formel zur Diskussion gestellt. ${ }^{2}$

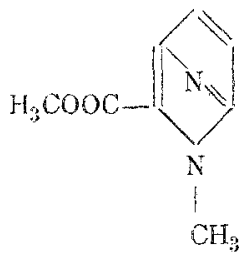

Um die Struktur des Ricinins einer Klärung entgegenzuführen, schien es uns vor allem notwendig, die Konstitution der Abbauprodukte $\mathrm{C}_{6} \mathrm{H}_{7} \mathrm{O}_{2} \mathrm{~N}$ (Maquenne und Philippe) und $\mathrm{C}_{7} \mathrm{H}_{9} \mathrm{O}_{2} \mathrm{~N}$ (Winterstein und Schüler) zu ermitteln. War die erstere Verbindung tatsächiich ein $N$-Methyloxypyridon, so wäre es möglich gewesci, dieselbe nach Maquenne und Philippe mittels Phosphorpentachlorid in das entsprechende Dichlorpyridin zu überführen und den so erhaltenen Körper mit einem Dichlorpyridin von bekannter Konstitution zu identifizieren. Nun sind aber die Dichlor-

1. Beuno Böttcher, Ber, der Deutschen Chem. Ges. 51, 673 (1918):

- Siche ferner die Formel von J. Keller, Dissertation. ZWich, 1915. 
pyridine zumeist schwer zugängliche Substanzen. Auch ist das aus dem Körper $\mathrm{C}_{6} \mathrm{H}_{7} \mathrm{O}_{2} \mathrm{~N}$ erhaltene Dichlorpyridin eine flüssige Verbindung, so daß eine zum sicheren Vergleiche notwendige gute Charakterisierung derselben durch Darstellung von krystallisierten Abkömmlingen wegen der schwach basischen Natur solcher Stoffe schwer möglich erscheint. Wir haben daher diesen naheliegenden Weg als wenig aussichtsvoll nicht in Angriff genommen.

Unter der Annahme, daB in der Verbindung $\mathrm{C}_{6} \mathrm{H}_{7} \mathrm{O}_{2} \mathrm{~N}$ ein N-Methyloxypyridon vorlag, wären im Ganzen folgende Pyridinabkömmlinge möglich gewesen.<smiles>Cn1cccc(O)c1=O</smiles><smiles>Cn1c(O)cccc1=O</smiles><smiles>Cn1ccc(O)cc1=O</smiles><smiles>Cn1ccc(=O)cc1O</smiles><smiles>Cn1cc(O)ccc1=O</smiles>

III<smiles>Cn1ccc(=O)c(O)c1</smiles>

Marl nätte diese Verbindungen det Reihe nach darstellen und mit dem aus dem Ricinin erhaltenen Produkt $\mathrm{C}_{6} \mathrm{H}_{7} \mathrm{O}_{2} \mathrm{~N}$ vergleichen können. Es muBte aber von einer derartigen Bearbeitung abgesehen werden, weil die meisten der oben genannten Stoffe nicht leicht darstellbare Verbindungen sind.

Wir haben nun aus Gründen, die hier in Kürze nicht wiedergegeben werden können, die Synthese der Verbindung II, die eventuell mit $\mathrm{V}$ tautomer sein konnte, in Angriff genommen.

Zunächst versuchten wir die Darstellung aus Chelidonsäure gemäb der Reaktionstolge<smiles>Cn1c(C(=O)O)cc(=O)cc1C(=O)O</smiles> 


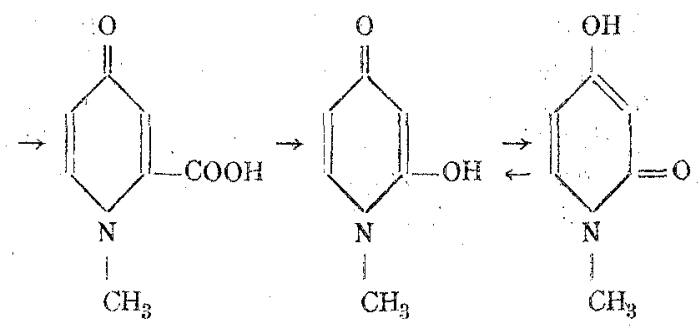

Diese Versuche scheiterten schon an dem. Umstande, dafs die $N$-Methylchelidamsäure keine leichte stufenweise Abspaltuing der einen Carboxylgruppe unter Bildung einer Monocarbonsäure erkennen ließ.

Wir stellten nun das von $G$. Errera durch Kondensation von Acetondicarbonsäureester, Orthoameisensäureester mit Ammoniak und nachherigem Behandeln mit Salzsäure erhaltene 2,4-Dioxypyridin her, überführten dasselbe in den $O$-Dimethyläther und behandelten dann mit Jodmethyl. Hierbei bildete sich jedenfalls zunächst das Jodmethylat, das aber durch spontane Abspaltung von Jodmethyl infolge Ablösung von Jod vom Stickstoff und Methyl von einer der beiden Methoxylgruppen in ein Pyridon überging, welches nach der Analyse und den vorgenommenen Umwandlungen eine Jer beiden nachfolgenden Formeln VII oder VIll haben konnte.

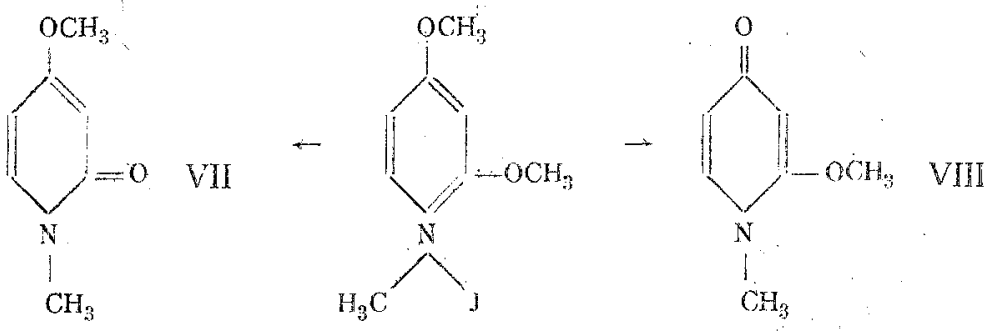

Diese Verbindung war nun in allen Eigenschaften identisch mit dem Körper $\mathrm{C}_{7} \mathrm{H}_{9} \mathrm{O}_{2} \mathrm{~N}$, welcher von $\mathrm{E}$. Winterstein und seinen Mitarbeitern ${ }^{2}$ durch Erhitzen von Ricinin mit $57 \cdot 4$ prozentiger Schwefelsäure auf $140^{\circ}$ erhalten worden war. Der Schmelzpunkt beider Verbindungen lag bei 113 bis $114^{\circ}$ und auch das Gemisch schmolz bei derselben Temperatur. Die Pikrate und Pikrolonate beider Körper waren ebenfalls völlig gleich.

Interessant war nun, daß diese Verbindung durch Erhitzen mit Salzsäure im Rohr unter Entmethylierung in einen bei $\$ 69$ bis $170^{\circ}$ schmelzenden Körper überging, welcher sich mit dem von Maquenne und Philippe aus Ricinin und Salzsäure bei $140^{\circ}$ erhaltenen Produkt $\mathrm{C}_{6} \mathrm{H}_{7} \mathrm{O}_{2} \mathrm{~N}$ identisch erwies.

1 G. Errera, Ber. der Deutschen Chem. Ges. 31, 1682 (1898).

2 E. Winterstein, J. Keller and A, B. Weinhagen, Arch der Wham. 255, 524 (1917). 
Durch die von uns vorgenommene Synthese ist aber sichergestellt, daß dem Körper $\mathrm{C}_{6} \mathrm{H}_{7} \mathrm{O}_{2} \mathrm{~N}$ eine der beiden folgenden Formeln zukommen muB.
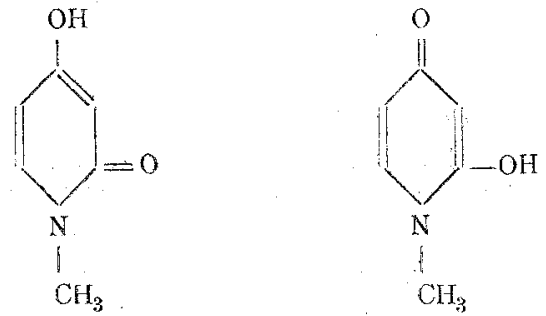

Da der von Winterstein, Keller und Weinhagen erhaltene Körper $\mathrm{C}_{7} \mathrm{H}_{9} \mathrm{O}_{2} \mathrm{~N}$ nach unseren Feststellungen der Methyläther einer der beiden obigen Verbindungen ist und die Methoxylgruppe in dieser Base jedenfalls an derselben Stelle des Pyridinkernes sitzt wie im Ricinin, war es für die Ermittlung der Konstitution dieses Alkaloids von großer Wichtigkeit festzustellen, ob der Methoxylrest in der Verbindung $\mathrm{C}_{7} \mathrm{H}_{9} \mathrm{O}_{2} \mathrm{~N}$ an der Stelle 2 (Formel VIII) oder an 4 (Formel VII) sich befindet. Wir versuchten zunächst durch die von van de Moer beim Cytisin aufgefundene Reaktion, aie auch dem $N$-Methyl-a-pyridon ${ }^{1}$ zukommt, zu ermitteln, ob in der untersuchten Verbindung ein $\alpha$ - oder ein $\gamma$-Pyridon vorlag. Die mit $N$-Methyl- $\alpha$-pyridon, $N$-Methyl- $\gamma$ pyridon und dem Körper $\mathrm{C}_{7} \mathrm{H}_{9} \mathrm{O}_{2} \mathrm{~N}$ vorgenommenen Versuche machten es ziemlich wahrscheinlich, daß die letztere Verbindung die Konstitution eines $N$-Methyl- $\gamma$-methoxy- $\alpha$-pyridons (VII) besitzt.

Einen sicheren Beweis für diese Annahme lieferte das Studium der Siedepunkte der in Betracht kommenden Körper: Während das $N$-Methyl- $\alpha$-pyridon bei $126.5^{\circ}$ und $14.5 \mathrm{~mm}$ siedete, destillierte das isomere $N$-Methyl- $\gamma$ pyridon etwa $100^{\circ}$ höher, nämlich bei 223 bis $224^{\circ}$ und $15 \mathrm{~mm}$. Eine ähnliche Differenz beobachtete der eine von uns bei den $N$-Methylchinolonen, indem das a.Chinolon bei $185^{\circ}$ und $13 \mathrm{~mm}^{2}$ und das $\gamma$-Chinolon bei 259 bis $261^{\circ}$ und $16 \mathrm{~mm}$ (Thermometer bis $40^{\circ}$ im Dampf) überging. Ist nun der Körper $\mathrm{C}_{7} \mathrm{H}_{9} \mathrm{O}_{2} \mathrm{~N}$ ein $\alpha$-Methoxy- $N$-methyl-\%-pyridon, so war zu erwarten, daß der Siedepunkt dieser Verbindung höher liegt als der des $N$-Methyl- $\gamma$-pyridons, weil der Ersatz von Wasserstoff gegen die Methoxylgruppe stets Erhöhung des Siedepunktes hervorruft. $\mathrm{Da}$ aber die Verbindung $\mathrm{C}_{7} \mathrm{H}_{3} \mathrm{O}_{2} \mathrm{~N}$ etwa $60^{\circ}$ niedriger siedete als das $N$-Methyl- $\gamma$-pyridon, nämich bei 161 bis $162^{\circ}$ und $16 \mathrm{~mm}$, so erscheint es gewi $\beta$, daß hier kein substituiertes $\gamma$-Pyridon, sondern ein a-Pyridon vorliegen muß. Die hier auftretende Siedepunktserhöhung beim Ersatz von Wasserstoff durch Methoxyl

1 E. Späth, Monatshefte für Chemie, 40, 26 (1919).

2 Monatshefte für Chemie, 40, 128 (1919). 
beträgt gegen das $N$-Methyl- $\alpha$-pyridon etwa $35^{\circ}$, was bei einem Körper von einem derartigen Siedepunkt entsprechend erscheint. Der daraus durch Verseifung erhaliene Körper $\mathrm{C}_{6} \mathrm{H}_{7} \mathrm{O}_{2} \mathrm{~N}$, der auch aus dem Ricinin entsteht, ist daher das $N$-Methyl-q-oxy- $\alpha$-pyridon, wobei über eine Tautomerie mit der Verbindung $V$ nichts ausgesagt werden kann.

Durch die hier beschriebene Synthese und die dann getroffene Auswahl zwischen den beiden möglichen Verbindungen ist die Konstitution des Ricinin-Abbau-Produktes $\mathrm{C}_{7} \mathrm{H}_{9} \mathrm{O}_{2} \mathrm{~N}$ als $\gamma$-Methoxy$N$-methyl-a-pyridon sicher ermittelt. Es ist nun die Frage, welche Schlüsse betreffs der Konstitution des Ricinins durch diese Feststellung: gezogen werden können.

Zunächst ersieht man, daß das Ricinin nicht der Methylester einer Carbonsäure sein kann, wie von den meisten Bearbeitern" dieses Gebietes angenommen worden ist, sondern daß die Methoxylgruppe dieses Alkaloids direkt an einem Pyridinkern in $\gamma$-Stellung zum Stickstoffatom sitzen mup. Die leichte Verseifbarkeit der Methoxylgruppe des Ricinins, die einen Carbonsäuremethylester vortäuschte, kann nur durch Änderung des Charakters des Pyridinkomplexes infolge Angliederung eines neuen Ringes erklärt werden, weil das Abbauprodukt dieses Alkaloids, welches noch den Methoxylrest enthält, dieselbe große Beweglichkeit dieser Gruppe nicht mehr zeigt.

Das $\gamma$-Methoxy- $N$-methyl-a-pyridon entsteht aus dem Ricinin gemä $\beta$ der folgenden Reaktion:

$$
\mathrm{C}_{8} \mathrm{H}_{8} \mathrm{O}_{2} \mathrm{~N}_{2}+2 \mathrm{H}_{2} \mathrm{O}=\mathrm{C}_{7} \mathrm{H}_{9} \mathrm{O}_{2} \mathrm{~N}+\mathrm{NH}_{3}+\mathrm{CO}_{2} \text {. }
$$

Nachdem das Ricinin mit verdünnten Säuren kaum Salze biluet, darf das als Ammoniak abgespaltene Stickstoffatom dieses Alkaloids keinen merklichen basischen Charakter mehr besitzen.

Es lag daher nahe, an ein Säureamid zu denken, wodurch auch noch die leichte Ablösung von Ammoniak beim Erhitzen von Ricinin mit Salzsäure wenigstens nach einer Seite hin erklärt werden konnte. Betreffs des Kohlenstoffatoms des abgespaltener Kohlendioxyds ist es klar, daß jedenfalls ein Sauerstoffatom an demselben vorhanden sein muß, falls man nicht die unwahrscheinliche Annahme machen wollte, daß ein Cyanrest im Ricinin vorkommt. Da ferner die beiden Sauerstoffatome des Ricinins durch die Methoxylgruppe und durch das am abspaltbaren C-Atom befindliche Sauerstuffatom mit Beschlag belegt sind, der $\alpha$-Pyridonsauerstoff im Ricinin selbst infolge Mangels an den charakteristischen Reaktionen der $\alpha$-Pyridone sicher nicht vorkommt, ist es zweitellos, daß das Kohlenstoffatom, an welchem nach der Spaltung der $\boldsymbol{x}$-Pyridonsauerstoff hängt, mit zwei Valenzen durch den ablösbaren Stickstoff abgesättigt sein mul3.

I Siehe dagegen Dissertation, J. Keller, Zürich 1915. 
Fassen wir alle Resultate und Überlegungen zusammen, so erhält man als Ergebnis derselben folgende zwei mögliche Konstitutionen des Ricinins.

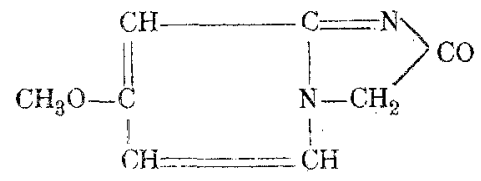

A

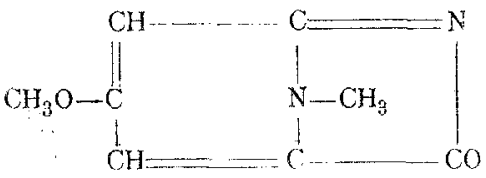

$\mathrm{B}$

Sie erklären zwanglos die Bildung des 4-Oxy- $N$-methyl$x$-pyridons und seines $O$-Methyläthers durch Ethitzen von Ricinin mit Salzsäure, beziehungsweise mit Schwefelsäure. Zunächst wird hierbei die Säureamidbindung gelöst und es entsteht eine Ketimidcarbonsäure, die dann, infolge der zumeist leichten Hydrolyse der Ketimide unter Wasserstoffaufnahme in eine Ketonsäure und Ammoniak übergeht. Schließlich wird Kohlendioxyd und eventuell Methylchlorid abgespalten und es entsteht das 4-Oxy- $N$-methyla-pyridon oder sein Methyläther. Die Reihenfolge der Spaltung könnte auch eine andere sein, als hier angegeben ist.

Die angeführten Formeln sind auch ein Hinweis auf den schwach basischen Charakter des Ricinins, seine Unlöslichkeit in verdünnten Laugen und den Mangel an Farbenreaktionen mit Eisenchlorid.

Unsere Formeln enthalten den Glyoxalinkern, der schon von Br. Böttcher infolge Auftretens der Weidelschen Reaktion und der leichten Abspaltung von Ammoniak angenommen worden ist.

Ist der Glyoxalinkern schwerer reduzierbar, wie öfters beobachtet worden ist, so würde sich ergeben, daß bei der katalytischen Reduktion des Ricinins, wie Winterstein und seine Schüler feststellten, vor allem ein Tetrahydroprodukt entsteht.

Die Formel B enthält die im Ricinin nachgewiesene Methylimidgruppe, doch auch die. Konstitution A läßt den' Nachweis einer Methylimidgruppe voraussehen, da sie durch Abspaltung von Kohlendioxyd nach Öffnung des Glyoxalinringes gebildet werden kann.

Durch die vorliegende Arbeit ist festgestellt, daß die bisher angenommenen Formeln des Ricinins unrichtig sind. Es ist auch gelungen, auf Grund der durch Synthese vorgenommenen Aufklärung der beiden Abbauprodukte des Ricinins in den Aufbau dieses Alkaloids so weit einzudringen, daß nur noch zwei Formeln möglich erscheinen. Wir werden versuchen, durch die Synthese des Ricinins eine sichere Entscheidung $\mathrm{zu}$ treffen. 


\section{Experimentelles.}

\section{Versuch der Synthese der Abbauprodukte über die Chelidonsäure.}

Die Darstellung der Chelidonsäure erfolgte nach Claisen ${ }^{k}$ und wurde so leicht in guter Ausbeute erhalten. Diese Säure wurde nach Lieben und Haitinger ${ }^{2}$ durch Erhitzen mit wässerigem Methylamin unter Ersatz von Sauerstoff gegen den Rest $\mathrm{NCH}_{3}$ in die Methylchelidamsäure übergeführt. Der Schmelzpunkt lag bei

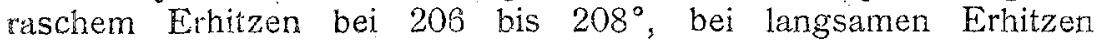
wesentlich tiefer. Die Ausbeute war $2 \cdot 5 \mathrm{~g}$ aus $5 \mathrm{~g}$. Chelidonsäure.

Wir versuchten nun, die eine Carboxylgruppe der $N$-Methylchelidamsäure abzuspalten und wollten dann die andere durch den Hofmannschen Abbau des Amids in die Amino- und dann in die Hyaroxylgruppe umwandeln.

Die Abspaltung einer Carboxylgruppe aus der $N$-Methylchelidamsäure gelang nicht durch Erhitzen der freien Säure. Wie ein quantitativer Versuch zeigte, spaltete die $N$-Methylchelidamsäure schon durch halbstündiges Erhitzen auf $160^{\circ} 2$ Moleküle Kohlendioxyd ab.

'Hierauf erhitzten wir das Mononatriumsalz der $N$-Methyichelidamsäure auf verschiedene Temperaturen, um die Bildung der Monocarbonsäure zu bewirken. Doch auch hier wurde kein günstiges Ergebnis erzielt.

Schließlich stellten wir das krystallisierte Monosilbersalz der $N$-Methylchelidamsäure her und behandelten dasselbe mit Jodmethyl. Doch war die Ausbeute an Estersäure, welche ein besseres Verhalten bei der Kohlendioxydabspaltung erhoffen ließ, schlecht.

Jedenfalls liegt bei der Chelidonsäure die Tendenz vor, beide Carboxylgruppen leicht abzuspalten. Da zu erwarten war, daß auch die weiter noch vorzunehmenden Reaktionen unerwünscht verlaufen würden, wurde von einer Vertiefung der beschriebenen orientiereriden Versuche abgesehen.

\section{Synthese der Abbauprodukte des Ricinins.}

Wir stellten zunächst das 2,4-Dioxypyridin nach dem Verfahren von G. Errera ${ }^{3}$ her. Der Schmelzpunkt lag bei 260 bis $261^{\circ}$, während Errera 260 bis $265^{\circ}$ angibt.

Zur Gewinnung des $O$ Dimethyläthers dieser Verbindung wurden $2 \cdot 77 \mathrm{~g} 2$, 4-Dioxypyridin in $250 \mathrm{~cm}^{3} \quad 1 / 5$-norm.-Natronlauge gelöst und mit $8 \cdot 49 \mathrm{~g}$ Silbernitrat in wenig Wasser unter Um-

1 L. Claisen, Ber. der Deutschen Chem. Ges, 24, 111 (1891).

L Leben und Haitinger, Monatshefte für Chemie, 6, 293 (1885)

3 (x, Ervera, Ber, der Deutschen Chem. Ges., 31, 1682 (1898). 
schütteln versetzt. Sogleich schied sich ein weiBliches Silbersalz aus, welches durch etwas Silberoxyd bräunlich gefärbt war. Zur vollständigen Umsetzung wurde eine Stunde auf der Maschine geschüttelt, dann abgesaugt, mit etwas Wasser gewaschen und im Vakuum getrocknet. Ausbeute $8 \cdot 2 \mathrm{~g}$.

Das Silbersalz wurde mit $50 \mathrm{~cm}^{3}$ absolutem Äthyläther übergossen und mit $7 \cdot 25 \mathrm{~g}$ Jodmethyl über Nacht stehen gelassen. Der filtrierten Lösung wurde durch Schütteln mit verdünnter Salzsäure der gebildete Dimethyläther entzogen und so eine Trennung desselben vom überschüssigen Jodmethyl bewirkt. Die sauere wässerige Lösung wurce alkalisch gemacht und dann durch Wasserdampf der in Freiheit gesetzte Dimethyläther übergetrieben. Durch nochmaliges etwa zwölfstündiges Einwirken von Jodmethyl auf das Gemisch von Jodsilber und das Silbersalz des 2, 4-Dioxypyridins wurde eine weitere Menge des Dimethyläthers in gleicher Weise erhalten. Die Gesamtausbeute des durch Äther ausgeschüttelten Produktes war $1.56 \mathrm{~g}$.

Zur Charakterisierung wurde das Pikrolonat hergestellt.

$0.2 \mathrm{~g}$ des Dimethyläthers wurden mit einer heißen Lösung von $0.34 \mathrm{~g}$ Pikrolonsäure in wenig heißem Äthylalkohol versetzt. Beim Verdünnen mit Wasser schied sich das Pikrolonat in Krystallen vom Schmelzpunkt 144 bis $146^{\circ}$ aus. Durch nochmaliges Lösen in heißem Alkohol und Fällen mit Wasser stieg der Schmelzpunkt auf 148 bis $149^{\circ}$. Der Schmelzpunkt ist etwas abhängig von der Geschwindigkeit des Erhitzens.

Die Methoxylbestimmung ist ein wenig zu niedrig, doch ist hierdurch ein Zweifel an der Zusammensetzung nicht gegeben. Vielleicht errährt der Dimethyläther, der, wie im folgenden gezeigt wird, äußerst leicht $N$-Methylpyridon gibt, bei der Methoxylbestimmung eine partielle Umlagerung.

$0.0812 \mathrm{~g}$ gaberr nạch $\mathrm{Z}$ eise $1.0 .0863 \mathrm{~g} \mathrm{Ag} \mathrm{J}$.

Gef: : $\mathrm{OCH}_{3} 14 \cdot 04 \%$;

ber. : für $\mathrm{C}_{15} \mathrm{H}_{11} \mathrm{O}_{5}\left(\mathrm{OCH}_{3}\right)_{2} \mathrm{~N}_{5} 15: 38 \% \mathrm{OCH}_{3}$.

Zur Darstellung: des Pyridons wurden $0.8 \mathrm{~g}$ des $O$-Dimethyläthers des 2, 4-Dioxypyridins mit $2 g$ Jodmethyl im verschlossenen Kölbchen bei Zimmertemperatur 3'Tage stehen gelassen. Schon nach mehreren Stunden schied sich eine Krystallmasse aus, deren Menge zunahm, aber am Ende der Einwirkung wieder geringer geworden war. Es wurde dann die Flüssigkeit von den noch vorhandenen Krystallen, abgegossen und mit wenig Ather nachgewaschen. Die krystallinische Substanz wurde im Vakuum getrocknet. Die Erwartung, daß in dieser Verbindung gemäß der vorgenommenen Umsetzung ein Jodmethylat vorliege, mußte fallen gelassen werden, weil nach dem Lösen in Wasser und Versetzen mit Silbernitrat keine Fällung von Jodsilber eintrat. Nach dem Trocknen bei $100^{\circ}$ schmolz die Verbindung scharf bei 113 bis 
$114^{\circ}$. Der Schmelzpunkt lag nach dem Umlösen aus wenig Äther bei derselben Temperatur. Das abgegossene flüssige Reaktionsprodukt hinterlieb beim Einengen gleichfalls Krystalle vom Schmelzpunkt 113 bis $114^{\circ}$ nach dem Trocknen bei $100^{\circ}$. Wie der Mischschmelzpunkt erwies, ist es dieselbe Verbindung. Die Ausbeute ist fast quantitativ.

Auf Grund der durchgeführten Reaktionen und der Methoxyibestimmung liegt der $O$-Methyläther eines $O x y-N$-Methyl- $x_{-}$oder y-pyridons vor.

$0.0684 \mathrm{~g}$ gaben nach Zeise $0.1162 \mathrm{~g} \mathrm{AgJ}$.

Gef.: $\mathrm{OCH}_{3} 22 \cdot 37 \%$;

ber.: für $\mathrm{C}_{6} \mathrm{H}_{6} \mathrm{O}\left(\mathrm{OCH}_{3}\right) \mathrm{N} 22 \cdot 30 \% \mathrm{OCH}_{3}$.

Die Verseifung erfolgte langsamer als bei einer normalen Methoxylbestimmung. Es wurde solange erhitzt, bis eine frische Beschickung von Silbernitrat beim Verdünnen mit Wasser keine Fällung mehr gab.

Etwas von dieser Verbindung wurde auch bei der Einwirkung von Jodmethyl auf das Silbersalz des 2, 4-Dioxypyridins erhalten. Der wässerige Auszug des Sibersalzes gab nach dem Versetzen mit Lauge und Ausschütteln mit Chloroform $0.37 \mathrm{~g}$ des bei 113 bis $114^{\circ}$ schmelzenden Körpers.

Diese Verbindung ist nun identisch mit der Substanz, welche Winterstein, Keller und Weinhagen ${ }^{x}$ beim Erhitzen von Ricinin mit $37 \cdot 4$ prozentiger Schwefelsäure auf $140^{\circ}$ erhalten haben.

Wir stellten, um diese Base mit unserem synthetischen Körper vergleichen zu können, Ricinin selbst dar. Das Material hierfür waren Preßrückstände von Ricinussamen, die wir von der Firma Fratelli Cavallo, Mailand, bezogen. Die Isolierung des Alkaloids erfolgte nach den Angaben von Winterstein und seinen Schülern. Das rohe, braun gefärbte Ricinin haben wir durch Umlösen aus einem Gemisch von 15 Teilen Xylol und 10 Teilen Amylalkohol sofort weiß und vom richtigen Schmelzpunkt erhalten.

Durch Erhitzen des Ricinins mit $57 \cdot 4$ prozentiger Schwefelsäure erbielten wir den von Winterstein und Mitarbeitern beschriebenen Körper $\mathrm{C}_{7} \mathrm{H}_{9} \mathrm{O}_{2} \mathrm{~N}$. Die Reinigung des Rohproduktes findet am leichtesten dadurch statt, $d a \beta$ man alkalisch macht und dann mit Chloroform ausschüttelt. Die saueren Produkte bleiben in der Lauge gelöst, während der gesuchte Körper in das Chloroform geht. Die so erhaltene Substanz schmolz zunächst bei 109 bis $111^{\circ}$, nach dem Umlösen aus Äther bei 113 bis $114^{\circ}$. Der Mischschmelzpunkt dieses Körpers mit unserem synthetischen Produkt lag bei derselben Temperatur. Diese Verbindungen sind daher identisch, was noch durch Darstellung von Abkömmlingen erhärtet wurde. 
Zur Darstellung des Pikrates der aus dem Ricinin erhaltenen Verbindung wurden $0.08 \mathrm{~g}$ in etwas Wasser gelöst und mit einer heißen Lösung von $0 \cdot 145 \mathrm{~g}$ Pikrinsäure in wenig Wasser versetzt. Sogleich schied sich das Pikrat in langen gelben verfilzten Nadeln vom Schmelzpunkt 154 bis $155^{\circ}$ rein ab. Die Ausbeute war $0 \cdot 2 \mathrm{~g}$.

Das aus dem synthetischen Produkt erhaltene Pikrat schmolz gleichfalls bei 154 bis $155^{\circ}$ und gab nach dem Vermischen mit der aus dem Ricinin erhaltenen Verbindung keine Depression des Schmelzpunktes.

Die Methoxylbestimmung stimmt auf die erwartete Verbindung. Sie ist noch schwerer verseifbar als die freie Base. Wir haben die Methoxylbestimmung auf viele Stunden ausgedehnt und bei neuer Beschickung mit Silbernitrat solange fortgesetzt, bis nach mehrstündigem Erhitzen und Aufarbeiten keine Fällung von Silberjodid mehr erinalten wurde.

$0.0963 \mathrm{~g} g$ gaben nach $Z$ eisel insgesant $0.0618 \mathrm{~g} \mathrm{AgJ}$.

Gef.: $8.51 \% \mathrm{OCH}_{3}$;

ber.: für $\mathrm{C}_{12} \mathrm{H}_{9} \mathrm{O}_{8}\left(\mathrm{OCH}_{3}\right) \mathrm{N}_{4} 8 \cdot 44 \% \quad \mathrm{OCH}_{3}$.

Auch das Pikrolonat ist eine charakteristische Verbindung.

$0.08 \mathrm{~g}$ des aus Ricinin erhaltenen Körpers $\mathrm{C}_{7} \mathrm{H}_{9} \mathrm{O}_{2} \mathrm{~N}$ wurden mit einer heißen Lösung von $0.152 g$ Pikrolonsäure in wenig Aikohol versetzt. Nach dem Erkalten wurde mit Wasser verdünnt und im Valrum eingeengt. Nach kurzer Zeit schieden sich gedrungene goldgelbe Krystalle aus, welche bei $126.5^{\circ}$ anscheinend unter Zersetzung schmolzen.

$0.0984 \mathrm{~g}$ gaben nach $Z$ eise $10.0572 \mathrm{~g}$ AgJ.

Ger: $7 \cdot 68 \% \quad \mathrm{OCH}_{3}$;

ber.: für $\mathrm{C}_{16} \mathrm{H}_{14} \mathrm{O}_{6}\left(\mathrm{OCH}_{3}\right) \mathrm{N}_{3} 7 \cdot 69 \% \mathrm{OCH}_{3}$.

Erhitzt man die synthetische Verbindung $\mathrm{C}_{7} \mathrm{H}_{9} \mathrm{O}_{2} \mathrm{~N}$ und dann die damit identische aus dem Ricinin erhaltene Substanz mit rauchender Salzsäure etwa 4 Stunden im Rohr auf $140^{\circ}$, so entsteht unter Verseifung der Methoxylgruppe ein Körfer $\mathrm{C}_{6} \mathrm{H}_{7} \mathrm{O}_{2} \mathrm{~N}$, welcher von Maquenne und Philippe aus Ricinin mit Salzsäure dargestellt worden war.

Alle drei so erhaltenen Verbindungen schmolzen nach dem Umlösen aus frisch destilliertem rohen Cumol, welches in der Hitze mäßig leicht, in der Kälte aber schwer löst, bei 1639 bis $170^{\circ}$. Auch die Gemische von je zwei Verbindungen hatten den gleichen Schmelzpunkt.

Währenđ̊ roch bei diesem Körper möglicherweise eine Tautomerie vorliegen kann, ist eine solche Umlagerungsmöglichkeit bei dem bei 113 bis $114^{\circ}$ schmelzenden Körper ausgeschlossen. Daher war es für die Konstitution des Ricinins von großer Wichtigkeit festzustellen, ob dieser Körper ein $\gamma$-Methoxy- $N$-methyl- $\alpha$-pyridon oder ein $\alpha$-Methoxy- $N$-methyl- $\gamma$-pyridon vorstellt. 
Zur Unterscheidung zwischen diesen beiden Formeln stellten wir zunächst einige Versuche mit der charakteristischen Farbenreaktion an, welche von van de Moer beim Cytisin aufgefunden worden war. Da, wie der eine von uns beobachtet hatte, dieselbe Reaktion auch bei $N$-Methyl-a-pyridonen auftritt, war zu erwarten, daßs unsere Verbindung die van de Moer'sche Reaktion nur dann geben werde, wenn in derselben ein $N$-Methyl-a-pyridon vorlag. Die Farbenreaktionen verliefen nun mit dem aus Ricinin erhaltenen Körper ganz ähnlich wie mit dem $N$-Methyl- $\alpha$-pyridon, während sie beim isomeren $N$-Methyl-\%-pyridon einen anderen Charakter hatte.

Wir arbeiteten unter Bedingungen, wie sie bereits durch den einen von uns in der Arbeit über das Cytisin beschrieben worden sind.

Beim $N$-Methyl- $\alpha$-pyridon erhält man mit Eisenchlorid die bekannte blutrote Färbung. Nach dem Hinzufügen von Wasserstoffsuperoxyd tritt in der Kälte eine langsame Farbenwandlung über Gelbgrün, Grün, Blaugrün in Blau ein. Erwärmt man nach dem Eintragen des Wasserstoffsuperoxyds, so tritt der Farbenumschlag in Blau tascher ein. Nach einigem Kochen wird die Lösung braun.

Das $N$-Methyl- $\gamma$-pyridon, das wir nach Lieben und Haitinger ${ }^{1}$ durch Destillation der $N$-Methylchelidamsäure darsteilten, gab mit Eisenchlorid bei gleichen Konzentrationsverhältnissen eine mehr gelbrote Färbung, die beim Hinzufügen von Wasserstoffsuperoxyd in Rotviolett überging. Die Färbung blieb lange bestehen und veränderte sich auch nicht bei kurzem Kochen.

Die Verbindung $\mathrm{C}_{7} \mathrm{H}_{9} \mathrm{O}_{2} \mathrm{~N}$ wurde mit Eisenchlorid blutrot und nach dem Eintragen von Wasserstoffsuperoxyd ziemlich rasch gelbgrün, grün, blaugrün und blau. Bei einigem Stehen wird die Färbung schmutziggrün und dann verschwindet sie ganz.

Sicheren Aufschluß gab der Vergleich der Siedepunkte. Das $N$-Methyl- $\alpha$-pyridon, das wir nach Decker ${ }^{2}$ herstellten, siedete bei $126.5^{\circ}$ (Thermometer bis $60^{\circ} \mathrm{im}$ Dampf) und $14.5 \mathrm{~mm}$, das $N$ Methyl- $\gamma$-pyridon destillierte bei 223 bis $224^{\circ}$ (Thermometer bis $30^{\circ} \mathrm{im}$ Dampf) und $15 \mathrm{~mm}$ und die Verbindung $\mathrm{C}_{7} \mathrm{H}_{9} \mathrm{O}_{2} \mathrm{~N}$ vom Schmelzpunkte 113 bis $114^{\circ}$ kochte bet 161 bis $162^{\circ}$ (Thermometer bis $30^{\circ}$ im Dampf) und $16 \mathrm{~mm}$.

Nach diesen Versuchen kann letztere Verbindung nur als

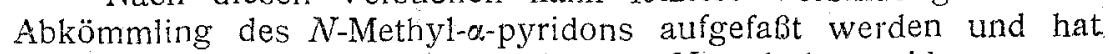
daher die Konstitution eines $\gamma$-Methoxy- $N$-methyl- $\alpha$-pyridons.

L Lieben und Haitinger, Monatshefte für Chemie, 6, 293 (1885).

2 Decker, Journ. für prakt. Chemie [2], 47, 29 (1893). 